\title{
Perdas reprodutivas e reconcepção em bovinos de corte segundo a idade ao acasalamento
}

\author{
[Reproductive disorders and reconception of beef cows according to of mating age] \\ C. Gottschall ${ }^{1}$, E. Ferreira ${ }^{2}$, L. Canellas $^{3,5}$, H.R. Bittencourt ${ }^{4}$ \\ ${ }^{1}$ Faculdade de Medicina Veterinária - ULBRA \\ Av. Farroupilha, 8001 \\ 90470-120 - Canoas, RS \\ ${ }^{2}$ Aluno de pós-graduação - UFRGS - Porto Alegre, RS \\ ${ }^{3}$ Aluno de graduação - ULBRA - Canoas, RS \\ ${ }^{4}$ Departamento de Estatística - PUCRS - Porto Alegre, RS \\ ${ }^{5}$ Bolsista da FAPERGS
}

\begin{abstract}
RESUMO
Avaliou-se o desempenho reprodutivo de novilhas de corte acasaladas aos 14 meses (14M), aos 24 meses $(24 \mathrm{M})$ e de vacas multíparas (V), totalizando 4.012 animais. As perdas reprodutivas (PR) foram de $19,3 \%, 11,6 \%$ e $7,6 \%$ para $14 \mathrm{M}, 24 \mathrm{M}$ e $\mathrm{V}$, respectivamente, sendo que $14 \mathrm{M}$ diferiu de $\mathrm{V}(\mathrm{P}<0,01)$. A ocorrência de distocia foi de $20,7 \%, 5,1 \%$ e $0,8 \%$ para as categorias $14 \mathrm{M}, 24 \mathrm{M}$ e $\mathrm{V}$, respectivamente, sendo que $14 \mathrm{M}$ diferiu de $\mathrm{V}(\mathrm{P}<0,01)$. Ocorreu maior mortalidade causada por distocia entre os animais do grupo $14 \mathrm{M}$ que entre os do grupo $\mathrm{V}(\mathrm{P}<0,01), 7,4 \%$ e $0,4 \%$, respectivamente. A reconcepção foi maior nos animais de $14 \mathrm{M}(85,3 \%)$ e V $(81,1 \%)$, comparada à dos de $24 \mathrm{M}(70,7 \%)(\mathrm{P}<0,01)$. A categoria que teve maior concentração de parição no primeiro período, 52,3\% (até 19/09), foi a de $24 \mathrm{M}(\mathrm{P}<0,01)$. Animais acasalados mais jovens tendem a apresentar maiores perdas reprodutivas e ocorrências de distocia.
\end{abstract}

Palavras-chave: bovino, acasalamento, desempenho reprodutivo, distocia

\begin{abstract}
Reproductive performances of beef heifers mated at 14 months of age (14M), at 24 months of age (24M) and pluriparous cows $(C)$, based on data from 4.012 animals were evaluated. The reproductive disorders were $19.3 \%, 11.6 \%$ and $7.6 \%$, respectively, at $14 M, 24 M$ and for $C ; 14 M$ was different from $C(P<0.01)$. The occurrence of dystocia was 20.7\%, 5.1\% and $0.8 \%$ for the $14 M, 24 M$ and $C$, respectively. The $14 M$ group was different from $C(P<0.01)$. The mortality caused by dystocia was higher for $14 M$ group $7.4 \%$ than for the $C$ group $0.4 \%(P<0.01)$, respectively. The reconception rate was higher for the $14 M(85.3 \%)$ and $V(81.1 \%)$ than for the $24 M$ group $(70.7 \%)(P<0.01)$. The category which concentrated the parturition in the first period (until 09/19) (52.3\%) was the 24M. Animals mated earlier tended to show high reproductive disorders and incidence of dystocia.
\end{abstract}

Keywords: bovine, mating, reproductive performance mating, dystocia

\section{INTRODUÇÃO}

$\mathrm{Na}$ busca para aumentar a eficiência reprodutiva e produtiva do rebanho bovino, pesquisadores vêm estudando diferentes sistemas de produção para otimizar a lucratividade de negócio. Um dos fatores que interferem significativamente na eficiência da pecuária de corte é o sistema de acasalamento adotado pela propriedade (Marshall et al., 1990; Machado et al., 2001).

Recebido em 12 de abril de 2007

Aceito em 11 de março de 2008

E-mail: carlosgott@cpovo.net 
Muitos trabalhos relataram que a diminuição da idade ao primeiro acasalamento é importante passo para alcançar maiores índices produtivos (Sawyer et al., 1991; Potter et al., 1998). Segundo Barcellos et al. (2003), a menor necessidade de novilhas para reposição, a eliminação de uma categoria etária de novilhas, a diminuição do intervalo entre gerações e a seleção precoce são razões que justificariam o acasalamento da novilha aos 14-15 meses de idade. Segundo Dias et al. (2004), desafiar as novilhas em idades mais jovens requer manejo adequado da fazenda, e essa prática poderá trazer benefícios com relação à seleção para precocidade sexual do rebanho, melhorando sua eficiência produtiva. Mas o acasalamento de animais muito jovens não traz somente vantagens. Existem fatores negativos e indesejáveis que também devem ser levados em consideração durante a escolha de determinado sistema de acasalamento. Short et al. (1994) apontaram algumas desvantagens em acasalar animais muito jovens, tais como maior exigência nutricional, maior custo alimentar, maior incidência de partos distocíacos, maiores perdas de terneiros e baixo peso ao desmame da primeira cria. Além disso, a incidência de perdas reprodutivas durante a gestação (como morte embrionária, por exemplo) é maior quando as fêmeas são acasaladas mais cedo (Erb e Holtz, 1958; Bellows e Short, 1994).

O objetivo do presente experimento foi avaliar o desempenho reprodutivo e a incidência de problemas reprodutivos em novilhas e vacas.

\section{MATERIAL E MÉTODOS}

O trabalho foi realizado a partir de informações oriundas de uma propriedade particular situada no município de Cristal, no estado do Rio Grande do Sul, obtidas entre 2001 a 2005. Foram analisadas informações de 4.012 fêmeas bovinas de corte, sendo 672 acasaladas aos 14/15 meses de idade (14M), 682 novilhas acasaladas aos $24 / 25$ meses de idade $(24 \mathrm{M})$ e 2.658 vacas acasaladas com idades entre três e 11 anos (V).

As categorias $14 \mathrm{M}$ e $24 \mathrm{M}$ foram submetidas a um manejo nutricional com o objetivo de atingirem cerca de $300 \mathrm{~kg}$ por ocasião da primeira estação de acasalamento. A alimentação dos animais do grupo 14M, após o desmame, foi baseada em pastejo em campo nativo acrescido de suplementação à base de concentrado com $18 \%$ de proteína bruta. No inverno, os animais foram alocados em pastagem cultivada de azevém (Lolium multiflorum), onde receberam suplementação à base de resíduos da indústria do arroz e sorgo com $12 \%$ de proteína bruta. A alimentação do grupo $24 \mathrm{M}$ foi baseada em pastejo em campo nativo e suplementação à base de resíduos da agroindústria do arroz durante o primeiro inverno. $\mathrm{O}$ grupo $\mathrm{V}$ foi manejado exclusivamente em campo nativo, com carga animal de aproximadamente 320 a $450 \mathrm{~kg} / \mathrm{ha}$, ajustada de acordo com a disponibilidade de forragem, variável conforme as condições climáticas e época do ano, objetivando uma condição corporal ao parto igual ou superior a 2,5 na escala de 1 a 5 (Lowman, 1976).

O manejo nutricional das fêmeas primíparas aos 24 meses de idade (grupo 14M) consistiu em pastejo em azevém no pré-parto de, no mínimo, 60 dias, objetivando uma condição corporal ao parto igual ou acima de três. A alimentação das primíparas aos 36 meses de idade (grupo 24M) foi à base de pastejo em campo nativo, com carga animal ajustada próxima aos $380 \mathrm{~kg} / \mathrm{ha}$, objetivando condição corporal ao parto igual ou superior a 2,5.

A primeira temporada de acasalamento das novilhas teve duração de 60 dias em média, iniciando-se no dia 19 de novembro e terminando no dia 19 de janeiro do ano seguinte. Para os animais com cria ao pé, a estação de acasalamento foi mais longa, de 90 dias em média, com início em 19 de novembro e término em 19 de fevereiro do ano seguinte. Os animais eram de origem britânica (Angus e Devon) com reduzido grau de sangue zebuíno (até 1/4).

O diagnóstico de prenhez, mediante palpação retal, foi realizado, em média, após 60 dias do término de cada estação de acasalamento. A data de parição foi dividida em quatro períodos, a fim de se obter uma distribuição da taxa de parição em função do tempo conforme cada categoria animal (histograma de parição). O primeiro período (1P) correspondeu às parições ocorridas até o dia 19/09 de cada ano. O segundo período (2P), às parições ocorridas entre $20 / 09$ e $05 / 10$, o terceiro período (3P), entre $06 / 10$ e $30 / 10$ e o quarto período (4P), entre 31/10 e 28/11. 
As variáveis analisadas foram: perdas reprodutivas (PR), resultantes do número de terneiros que não nasceram em relação ao total de fêmeas prenhes diagnosticadas na palpação retal - aqui foram incluídas mortalidade fetal, aborto, reabsorção fetal, morte por distocia e natimortos -; ocorrência de distocia; taxa de parição (TP), representada pelo número de terneiros nascidos vivos; e repetição de prenhez (RP). Para calcular os índices de distocia e morte por distocia, avaliaram-se apenas as fêmeas que entraram em processo de parto, ou seja, animais que apresentaram perdas reprodutivas foram excluídos do cálculo.

Os dados foram coletados no campo e posteriormente tabulados em planilha Microsoft Excel. A análise estatística foi realizada no pacote SPSS versão 13.0, consistindo de tabelas de freqüência simples e cruzadas e do teste quiquadrado (SPSS ..., 2004).

\section{RESULTADOS E DISCUSSÃO}

Na Tab.1, observam-se as perdas reprodutivas, segundo a categoria estudada.

As novilhas acasaladas aos 14 meses (14M) foram as que apresentaram maior ocorrência de partos distocíacos. Fontana (1993) também relatou maior incidência de distocia em novilhas Hereford acasaladas mais cedo em relação às acasaladas mais tardiamente. Schafhäuser Jr. et al. (2004) salientaram que novilhas acasaladas muito jovens podem apresentar maior incidência de distocia em razão do pequeno tamanho de sua área pélvica e do maior tamanho do terneiro ao nascer. Young (1968), na Austrália, relatou perdas reprodutivas de $17 \%$ para vacas de primeira cria e de apenas 3,5\% para ventres com mais de uma parição, atribuindo essa diferença, principalmente, à mortalidade dos terneiros decorrentes de partos distocíacos. Bellows (1968) relatou $43 \%$ e $16 \%$ de ocorrência de distocia para novilhas acasaladas aos $14 \mathrm{M} \mathrm{e}$ $24 \mathrm{M}$, respectivamente.

A categoria $14 \mathrm{M}$ apresentou maior taxa de perdas reprodutivas, resultado maior que o encontrado por Barros e Visintin (2001), cujas taxas de perdas por morte embrionária e fetal foram, em média, de $4,6 \%$ e $4,7 \%$ para vacas e novilhas zebuínas, respectivamente. Maior taxa de perda reprodutiva é relatada por Forar et al. (1995): 10,6\% entre novilhas e vacas de corte. De acordo com Erb e Holtz (1958), animais mais jovens, quando acasalados, tendem a apresentar maiores incidências de distúrbios na gestação, como, principalmente, morte embrionária.

Quando se trabalha com estação de monta fixa, como neste estudo, a informação da data de parição para o rebanho bovino é uma variável importante na pecuária de corte, pois, quanto mais cedo ocorrer o parto, mais tempo a vaca terá para voltar a ciclar até o início do próximo acasalamento, aumentando as chances de concepção na próxima estação de acasalamento. A Tab. 2 apresenta a distribuição da parição segundo os períodos e a categoria animal.

Tabela 1. Ocorrência de distocia, morte por distocia e perdas reprodutivas de fêmeas, de acordo com a categoria, durante os anos de 2001 a 2005

\begin{tabular}{|c|c|c|c|c|c|c|c|c|c|}
\hline & \multicolumn{3}{|c|}{ Vaca } & \multicolumn{3}{|c|}{ Novilha de 24 meses } & \multicolumn{3}{|c|}{ Novilha de 14 meses } \\
\hline & \multirow{2}{*}{ Total } & \multicolumn{2}{|c|}{ Incidência } & \multirow{2}{*}{ Total } & \multicolumn{2}{|c|}{ Incidência } & \multirow{2}{*}{ Total } & \multicolumn{2}{|c|}{ Incidência } \\
\hline & & $\mathrm{n}$ & $\%$ & & $\mathrm{n}$ & $\%$ & & $\mathrm{n}$ & $\%$ \\
\hline Distocia & 2455 & 20 & $0,8 \mathrm{a}$ & 603 & 31 & $5,1 \mathrm{ab}$ & 542 & 112 & $20,7 b$ \\
\hline Morte por distocia & 2455 & 10 & $0,4 a$ & 603 & 5 & $0,8 \mathrm{ab}$ & 542 & 40 & $7,40 \mathrm{~b}$ \\
\hline Perdas reprodutivas & 2658 & 203 & $7,6 \mathrm{a}$ & 682 & 79 & $11,6 a b$ & 672 & 130 & $19,3 b$ \\
\hline
\end{tabular}

Médias na mesma linha seguidas de letras diferentes diferem entre si $(\mathrm{P}<0,01)$ pelo teste qui-quadrado.

As fêmeas acasaladas aos 24 meses de idade (24M) foram as que pariram mais cedo. Entretanto, o fato de parir mais cedo não influenciou a reconcepção (Tab.3). Resultados semelhantes ao do presente experimento foram relatados por Marshall et al. (1990), que não observaram diferença no desempenho reprodutivo de fêmeas paridas nos primeiros 21 dias da estação de parição $(78,1 \%$ de prenhez) em relação às paridas entre o $22^{\circ}$ dia e o $42^{\circ} \quad(72,7 \%$ de prenhez). Resultados diferentes foram apresentados por Azeredo et al. (2007), que relatam maior taxa de reconcepção a favor das fêmeas paridas no início da estação de parição. 
Perdas reprodutivas e reconcepção...

Tabela 2. Distribuição de parição acumulada ao longo dos períodos, de acordo com a categoria, durante os anos de 2001 a 2005

\begin{tabular}{|c|c|c|c|c|c|c|c|c|}
\hline \multirow{3}{*}{ Categoria } & \multicolumn{8}{|c|}{ Período } \\
\hline & \multicolumn{2}{|c|}{$1 \mathrm{P}(19 / 09)$} & \multicolumn{2}{|c|}{$2 \mathrm{P}(05 / 10)$} & \multicolumn{2}{|c|}{$3 \mathrm{P}(30 / 10)$} & \multicolumn{2}{|c|}{$4 \mathrm{P}(28 / 11)$} \\
\hline & $\mathrm{n}$ & $\%$ & $\mathrm{n}$ & $\%$ & $\mathrm{n}$ & $\%$ & $\mathrm{n}$ & $\%$ \\
\hline Vaca & 894 & $33,6 \mathrm{a}$ & 1572 & 59,1 a & 2095 & $78,8 \mathrm{a}$ & 2455 & $92,4 \mathrm{a}$ \\
\hline Novilha de $24 \mathrm{M}$ & 357 & $52,3 \mathrm{~b}$ & 496 & $72,7 \mathrm{~b}$ & 594 & $87,1 \mathrm{~b}$ & 603 & $88,4 \mathrm{ab}$ \\
\hline Novilha de $14 \mathrm{M}$ & 249 & $37,1 \mathrm{ab}$ & 441 & $65,6 \mathrm{ab}$ & 539 & $80,2 \mathrm{ab}$ & 542 & $80,7 \mathrm{~b}$ \\
\hline Total & 1500 & & 2509 & & 3228 & & 3600 & \\
\hline
\end{tabular}

Médias na mesma coluna, seguidas de letras distintas, diferem entre si pelo teste qui-quadrado $(\mathrm{P}<0,01)$.

Tabela 3. Reconcepção conforme a categoria animal

\begin{tabular}{|c|c|c|c|}
\hline \multirow{2}{*}{ Categoria } & \multirow{2}{*}{ Total } & \multicolumn{2}{|c|}{ Reconcepção } \\
\hline & & $\mathrm{n}$ & $\%$ \\
\hline Vacas & 2658 & 2156 & $81,1 \mathrm{a}$ \\
\hline Novillas de 24 meses & 682 & 482 & $70,7 \mathrm{~b}$ \\
\hline Novilhas de 14 meses & 672 & 573 & $85,3 \mathrm{a}$ \\
\hline
\end{tabular}

Médias na mesma coluna seguidas de letras distintas, diferem entre si pelo teste qui-quadrado $(\mathrm{P}<0,01)$.

As categorias que apresentaram maior repetição de prenhez foram as $14 \mathrm{M}$ e as V. Lobato e Magalhães (2001) também mostraram que é possível acasalar novilhas aos 14-15 meses de idade, com parição aos 24-25 meses, sem efeitos negativos no processo reprodutivo subseqüente, quando se utiliza manejo alimentar de qualidade, rico em energia e proteína. Nesse estudo, os autores utilizaram pastagens melhoradas de ciclo hiberno-primaveril e atingiram taxas de prenhez de $93,1 \%$ e $86,2 \%$ para vacas primíparas aos 24 e 36 meses de idade, respectivamente. Rovira (1974) e Fahmy (1971) relataram taxas de repetição de prenhez em vacas primíparas aos 24 e 36 meses, respectivamente, de $82,2 \%$ e $75,6 \%$ e $88,3 \%$ e $77,4 \%$, sugerindo relação positiva entre velocidade de crescimento e fertilidade.

$\mathrm{Na}$ Tab. 4, mostra-se o desempenho reprodutivo, expresso pela taxa de parição, dos animais pertencentes às diferentes categorias.

Tabela 4. Taxa de parição de fêmeas, de acordo com a categoria

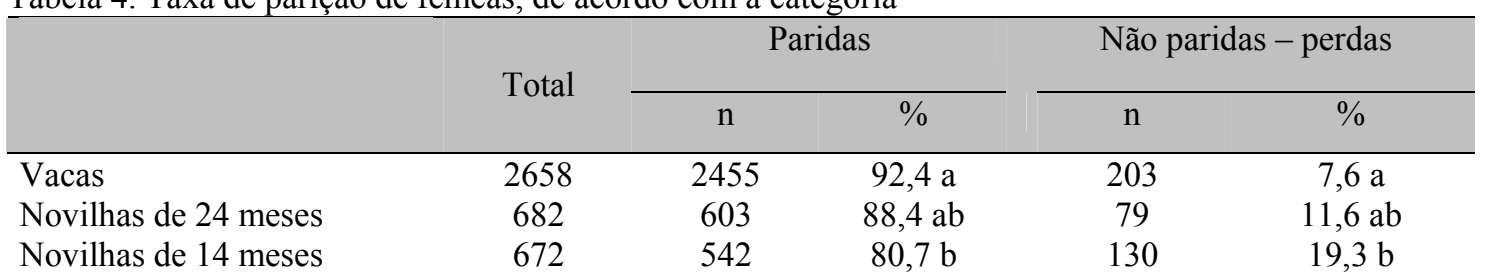

Médias na mesma coluna seguidas de letras distintas diferem entre si pelo teste do qui-quadrado $(\mathrm{P}<0,01)$.

Observa-se que a categoria $\mathrm{V}$ apresentou maior taxa de parição ao longo dos cinco anos. Diversos trabalhos também reportam à tendência de animais mais velhos atingirem melhor desempenho reprodutivo que animais mais jovens (Short e Bellows, 1971; Barcellos et al., 2006). Conforme Short et al. (1990), os requisitos nutricionais de novilhas acasaladas mais jovens são mais altos, priorizando a energia proveniente da dieta para o crescimento, em detrimento das funções reprodutivas. Tal fato explicaria o melhor desempenho reprodutivo (taxa de parição) por parte das vacas em relação às novilhas de 14 e 24 meses de idade.

\section{CONCLUSÕES}

A idade ao acasalamento influencia a reconcepção e as perdas reprodutivas em vacas e novilhas de corte. Embora novilhas acasaladas mais precocemente apresentem maiores perdas reprodutivas e menor taxa de parição, atendidas as necessidades nutricionais, o acasalamento mais precoce de novilhas poderá ser compensado pela maior taxa de repetição desta categoria. 


\section{REFERÊNCIAS BIBLIOGRÁFICAS}

AZEREDO, D.M ; ROCHA, D.C.; JOBIM, M.I.M. et al. Efeito da sincronização e da indução de estros em novilhas sobre a prenhez e o índice de repetição de crias na segunda estação reprodutiva Cienc. Rural, v.37, p.201-205, 2007

BARCELLOS, J.O.J.; COSTA, E.C.; SEMMELMANN, C.E.N. et al. Manejo nutricional da novilha até o primeiro acasalamento. In: SIMPÓSIO DE REPRODUÇÃO DE BOVINOS, 2., 2003, Porto Alegre. Anais...Porto Alegre, 2003. p.4-27.

BARCELLOS, J.O.J.; SILVA, M.D.; PRATES, E.R. et al. Taxas de prenhez em novilhas de corte acasaladas aos 18 e 24 meses de idade. Arq. Bras. Med. Vet. Zootec., v.58, p.1168-1173, 2006.

BARROS, B.J.P.; VISINTIN, J.A. Controle ultrasonográfico de gestações, de mortalidades embrionárias e fetais e do sexo de fetos bovinos zebuínos. Braz. J. V. Res. Anim. Sci., v.38, p.112-119, 2001 .

BELLOWS, R.A. Reproduction and growth in beef heifers. Artif. Insem. Dig., v.16, p.6-10, 1968.

BELLOWS, R.A.; SHORT, R.E. Reproductive losses in the beef industry. In: FIELDS, M.J.; SAND, R.S. (Eds). Factors affecting calf crop. Boca Raton: CRC Press, 1994. p.109-133.

DIAS, L.T.; FARO, L. EL; ALBUQUERQUE, L.G. Efeito da idade de exposição de novilhas à reprodução sobre estimativas de herdabilidade da idade ao primeiro parto em bovinos Nelore. Arq. Bras. Med. Vet Zootec., v.56, p.370-373, 2004.

ERB, R.E.; HOLTZ, E.W. Factors associated with estimated fertilization and service efficiency of cows. J. Dairy Sci., v.41, p.1541-1552, 1958.

FAHMY, M.H.; LALANDE, G.; HIDIROGLOU, M. Reproductive performance and growth of Shorthorn purebred and crossbred cows. Anim. Prod., v.13, p.7-14, 1971.

FONTANA, P.M.F. Distocia em novilhas Hereford com parição aos 24 meses de idade. 1993. $96 \mathrm{f}$. Dissertação (Mestrado) - Universidade Federal de Pelotas, Pelotas, RS

FORAR, A.L.; GAY, J.M.; HANCOCK, D.D. The frequency of endemic fetal loss in dairy cattle: a review. Theriogenology, v.43, p.989-1000, 1995.

LOBATO, J.F.P; MAGALHÃES, F.R. Comportamento reprodutivo de vacas primíparas aos 24 e aos 36 meses de idade. Arq. Fac. Vet. UFRGS v.29, p.139-146, 2001.
LOWMAN, B.G.; SCOTT, N.; SOMERVILLE, S. Condition scoring beef cattle. Edinburgh: Scotland College of Agriculture, 1976. 8p.

MACHADO, P.F.A; BERGMANN, J.AG; PEREIRA, J.C.C. et al. Predição da taxa de gestação de novilhas da raça Nelore acasaladas com um ano de idade. Arq. Bras. Med. Vet. Zootec., v.53, p.1-10, 2001.

MARSHALL, D.M.; MINQUIANG, W.; FREKING, B.A. Relative calving date of first-calf heifers as related to production efficiency and subsequent reproductive performance. J. Anim. Sci., v.68, p.18121817,1990

POTTER, L.; LOBATO, J.F.P.; MIELITZ NETTO, C.G. Produtividade de um modelo de produção para novilhas de corte primíparas aos dois, três e quatro anos de idade. Rev. Bras. Zootec., v.27, p.613-615, 1998.

ROVIRA, J. (Ed). Reproducción y manejo de los rodeos de cria. Montevideo: Hemisfério Sur, 1974. 293p.

SAWYER, G.D.; BARKER, D.J.; MORRIS, J. Performance of young breeding cattle in commercial herds in the south-west of Western Australia. Liveweight, body condition, conception and fertility in first calf heifers. Austr. J. Exp. Agric., v.31, p.443454, 1991

SCHAFHÄUSER JR, J.; MANCIO, A.B.; FONTES, C.A.C et al. Desempenho reprodutivo de novilhas com diferente grau de musculosidade. Rev. Fac. Zootec. Vet. Agro., v.10, p.2-19, 2004.

SHORT, R.E.; BELLOWS, R.H. Relationships among weight gains, age at puberty and reproductive performance in heifers. J. Anim. Sci., v.32, p.127131,1971

SHORT, R. E.; BELLOWS, R. H.; STAIGMILLER, R. B.; et al. Physiological mechanisms controlling anestrus and infertility in postpartum beef cattle. J. Anim. Sci., v.68, p.799-816, 1990

SHORT, R.E.; STAIGMILLER, R.B.; BELLOWS, R.A. et al. Breeding heifers at one year of age: biological and economic considerations. In: FIELDS, M.J.; SAND, R.S. (Eds). Factors affecting calf crop. Boca Raton : CRC Press, 1994. p.55-68.

SPSS Base 13.0. User's guide. Chicago: SPSS Inc., 2004. 712p.

YOUNG, J.S. Breeding patterns in commercial beef herds. I. Herd performance in New South Wales. Austr. Vet. J., v.44, p.350-356, 1968. 